\title{
Association of Various Anthropometric Indices with Sudomotor Dysfunction in Type 2 Diabetic Patients
}

\author{
Bekzod Odilov1,2, Danfeng Yu',2, Amir Muse Mohamud ${ }^{3,4}$, Ruxing Zhao ${ }^{2,5,6,7}$, Ying Zou',2, \\ Xinguo Hou ${ }^{1,2,5,6,7^{*}}$ \\ ${ }^{1}$ Department of Endocrinology, School of Medicine, Cheeloo College of Medicine, Shandong University, Jinan, China \\ ${ }^{2}$ Department of Endocrinology, Qilu Hospital of Shandong University, Jinan, China \\ ${ }^{3}$ Department of Nephrology, School of Medicine, Cheeloo College of Medicine, Shandong University, Jinan, Shandong, China \\ ${ }^{4}$ Department of Nephrology, Qilu Hospital of Shandong University, Jinan, China \\ ${ }^{5}$ Institute of Endocrine and Metabolic Diseases of Shandong University, Jinan, China \\ ${ }^{6}$ Key Laboratory of Endocrine and Metabolic Diseases, Shandong Province Medicine \& Health, Jinan, China \\ ${ }^{7}$ Jinan Clinical Research Center for Endocrine and Metabolic Diseases, Jinan, China \\ Email: ^houxinguo@sdu.edu.cn
}

How to cite this paper: Odilov, B., Yu, D., Mohamud, A.M., Zhao, R., Zou, Y. and Hou, X. (2022) Association of Various Anthropometric Indices with Sudomotor Dysfunction in Type 2 Diabetic Patients. Journal of Diabetes Mellitus, 12, 35-49. https://doi.org/10.4236/jdm.2022.121005

Received: December 23, 2021

Accepted: January 14, 2022

Published: January 17, 2022

Copyright $\odot 2022$ by author(s) and Scientific Research Publishing Inc. This work is licensed under the Creative Commons Attribution International License (CC BY 4.0).

http://creativecommons.org/licenses/by/4.0/ Open Access

\begin{abstract}
Aim: To investigate the relationship between sudomotor dysfunction and various body composition analysis indices in type 2 diabetic patients. $\mathrm{Me}$ thods. Between January 2016 and April 2021, 136 diabetic participants who had undergone body composition analysis (BCA) were recruited for this cross-sectional study. Sudomotor functions were assessed using SUDOSCAN, and participants were grouped into patients with normal (Group 1, $\mathrm{n}=51$ ), mildly reduced (Group $2, \mathrm{n}=46$ ) and severely reduced (Group $3, \mathrm{n}=39$ ) foot electrochemical skin conductance (FESC) levels. Results. The mean age was $60.4 \pm 10.1$ years, median diabetes duration was $12(6-19)$ years, and $52.2 \%$ of participants were males. Among BCA parameters, the significant differences were found in total fat (TF) $(p=0.023)$, percentage of TF $(\% \mathrm{TF})(\mathrm{p}=$ $0.025)$, percentage of android fat (\%AF) $(\mathrm{p}=0.048)$, fat mass (FM) in arms ( $\mathrm{p}$ $=0.016), F M$ in legs $(p=0.002)$, appendicular fat mass $(\mathrm{aFM})(\mathrm{p}=0.002)$, appendicular fat mass/body mass index $(\mathrm{aFM} / \mathrm{BMI})$ ratio $(\mathrm{p}=0.009)$ between three groups. In Spearman correlation analysis, FESC was correlated with RBC, ESR and homocysteine $(r=0.171, r=-0.190, r=-0.192, p<0.05)$, respectively. Multivariate linear regression analysis revealed that FM in arms, FM in legs, aFM and aFM/BMI ratio were independently associated with FESC even after adjustment for age, diabetes duration, WC, systolic BP, HbA1c, FPG, HOMA-IR, ESR, HDL-C, LDL-C, Total Cholesterol, ALT ( $\beta=$
\end{abstract}


$0.161,0.155,0.165,0.185, \mathrm{p}<0.05$, respectively). Conclusion: The progressive decline of sudomotor function is positively associated with loss of subcutaneous fat in arms and legs, suggesting that subcutaneous fat of extremities may be necessary to prevent DPN progression in type 2 diabetic patients.

\section{Keywords}

Diabetic Neuropathy, SUDOSCAN, Subcutaneous Fat, Sudomotor

Dysfunction, Anthropometric Parameters

\section{Introduction}

According to global estimation in 2019, diabetes had affected more than 463 million adults, mainly type 2 diabetics [1]. Diabetic peripheral neuropathy (DPN) is one of the leading diabetic complications in terms of prevalence [2] and diabetes-related medical costs [3], which has a negative impact on patients' quality of life [4]. Furthermore, future estimations related to diabetes are unfavourable, expecting that almost one-third of individuals living in 2050 will be affected by diabetes, and even worse, DPN will be present in nearly $50 \%$ of those patients [5].

On the other hand, sarcopenia-another global healthcare issue present in nearly 50 million people currently, is predicted to rise tenfold in 2050 [6]. Briefly, sarcopenia is a syndrome associated with a generalized and progressive decline of muscle mass and strength, resulting in reduced physical ability and life expectancy [7] [8]. Different tools have been implicated in muscle assessment in sarcopenia, including Computed tomography (CT), Magnetic resonance imaging (MRI), dual energy X-ray absorptiometry (DXA), etc. [9]. However, due to low cost and radiation risk, DXA is commonly applied in clinical practice for BCA [10]. Several studies [11] [12] have found that patients with type 2 diabetes mellitus (T2DM) have a higher risk for low appendicular muscle mass than non-diabetic individuals. Even more, a diabetes-sarcopenia association may be present in the very early stages of diabetes and may further progress due to diabetic complications, particularly nerve damage related to muscle atrophy [13] [14]. In addition to low muscle mass, diabetic neuropathy also contributes to the exacerbation of motor dysfunction in patients with sarcopenia [15]. Thus, early identification of both sarcopenia and diabetic neuropathy poses great importance.

Small fiber neuropathy (SFN) is considered an initial stage of DPN and is characterized by unmyelinated $\mathrm{C}$ fibers damage [16]. Although nerve conduction studies (NCS) and Michigan Neuropathy Screening Instrument (MNSI) questionnaires are mainly used in daily practice to diagnose DPN, and they are less effective and insensitive for SFN detection [17]. SUDOSCAN is a sensitive, 
inexpensive diagnostic tool for SFN detection through sudomotor nerve function evaluation [18] [19].

Associations between different diabetic complications and low muscle mass have been previously investigated [20] [21] [22]. However, a possible relationship between sudomotor dysfunction, which indicates SFN, and low muscle mass in subjects with type 2 diabetes remains undiscovered.

Our present study aimed to investigate the relationship between sudomotor dysfunction and various body composition analysis indices in type 2 diabetic patients.

\section{Method}

\subsection{Study Population}

Overall, 136 diabetic patients visited Qilu Hospital of Shandong University in January 2016 and April 2021 with available BCA results were recruited for the current cross-sectional study. T2DM diagnosis was following World Health Organization (WHO) 1999 criteria [23]. All subjects were categorized into three groups according to previous studies [24]:

Group 1: Subjects with normal $(>70 \mu \mathrm{S})$ foot electrochemical skin conductance (FESC) $(\mathrm{n}=51)$

Group 2: Subjects with mildly reduced $(50-70 \mu S)$ FESC $(n=46)$

Group 3: Subjects with severely reduced $(<50 \mu S)$ FESC $(n=39)$

Patients under 18 or over 80 years, those out of the $18-40 \mathrm{~kg} / \mathrm{m}^{2}$ body mass index (BMI) range, breastfeeding/pregnant, immobilized patients and patients with missing information in medical records were excluded. Moreover, patients using glucocorticoids, patients with arm/leg amputation, electrical implantable devices, those with type 1 diabetes or monogenic types of diabetes, cancer, history of seizures or epilepsy, severe vitamin B12 deficiency, Parkinson's disease, sciatic nerve lesion, hypothyroidism, advanced varices of lower extremities, end-stage renal disease (ESRD), severe hepatic disorders and those who abused alcohol (males with $\geq 140 \mathrm{~g} /$ week and females with $\geq 70 \mathrm{~g} /$ week alcohol consumption) [25] were also not enrolled in our study. Written informed consent has been provided by all participants before enrollment in the study. The Qilu hospital of Shandong University's ethical committee approved the study protocol.

\subsection{Data Collection}

Medical records were used to obtain data related to patient's demographics, medical history, lifestyle behaviours and current medication use, such as duration of diabetes, alcohol intake or smoking, present co-morbidities (hypertension, cardiovascular disease, dyslipidemia, osteoporosis) and complications (mainly, diabetic retinopathy). Anthropometric measurements including weight $(\mathrm{kg})$, height $(\mathrm{m})$, waist circumference $(\mathrm{WC})(\mathrm{cm})$, along seated blood pressure (BP) levels were obtained under standardized protocols, and BMI was also cal- 
culated before BCA measurement.

\subsection{Biochemical Evaluation}

The results of following biochemical parameters were collected: hemoglobin $(\mathrm{Hb})$, red blood cells (RBC), erythrocyte sedimentation rate (ESR), fasting plasma glucose (FPG), HbA1c, eGFR (calculated using CKD-EPI 2009 formula), homocysteine, uric acid, total bilirubin, alanine aminotransferase (ALT) and standard lipid profile with triglycerides, total cholesterol (TC), high-density lipoprotein cholesterol (HDL-C), low-density lipoprotein cholesterol (LDL-C). In addition to these measurements, various indices including lipid accumulation product (LAP), visceral adiposity index (VAI), Chinese VAI, Triglyceride-glucose index (TyG) and Homeostatic Model Assessment for Insulin Resistance (HOMA-IR) were calculated using specific formulas. Particularly, following formula was used for TyG index calculation: $\ln$ [fasting triglycerides $(\mathrm{mg} / \mathrm{dl}) \times$ fasting plasma glucose $(\mathrm{mg} / \mathrm{dl})]$ [26]. VAI calculation was based on gender-specific equations described by Amato et al. [27]: men: [WC/39.68 + $(1.88 \times \mathrm{BMI})] \times(\mathrm{TG} / 1.03) \times$ (1.31/HDL); women: [WC/36.58 + (1.89 × BMI) $\times(\mathrm{TG} / 0.81) \times(1.52 / \mathrm{HDL})$. LAP was calculated using the specific equation [28]: [WC $(\mathrm{cm})-65] \times[\mathrm{TG}$ $(\mathrm{mmol} / \mathrm{l})]$ for men; [WC $(\mathrm{cm})-58] \times[\mathrm{TG}(\mathrm{mmol} / \mathrm{l})]$ for women. CVAI was calculated according to published formula [29]: Males: CVAI $=-267.93+0.68 \times$ age $+0.03 \times \mathrm{BMI}+4.00 \times \mathrm{WC}+22.00 \times \log 10(\mathrm{TG})-16.32 \times$ HDL; Females: $\mathrm{CVAI}=-187.32+1.71 \times$ age $+4.23 \times \mathrm{BMI}+1.12 \times \mathrm{WC}+39.76 \times \log 10(\mathrm{TG})-$ $11.66 \times$ HDL. Previously mentioned formula was used to calculate HOMA-IR [30]: HOMA-IR $=$ fasting glucose $(\mathrm{mmol} / \mathrm{L}) \times$ fasting insulin $(\mu \mathrm{U} / \mathrm{mL}) / 22.5$.

\subsection{Body Composition Evaluation}

A qualified staff analysed the body composition analysis of all subjects using a HorizonTM DXA System (Hologic, Inc., Marlborough, MA, USA). Following body composition parameters were measured: percentage of android fat (\%AF), total fat (TF), percentage of TF (\%TF), total lean mass (TLM), fat (FM) and lean mass (LM) on both arms and legs. The sum of lean mass on arms and legs was defined as appendicular LM (aLM). aLM/BMI ratio and relative skeletal muscle index (RSMI) were also calculated using specific formulae [31] [32]. Additionally, we also proposed two novel body composition parameters: appendicular fat mass $(\mathrm{aFM})=\mathrm{FM}$ in arms + FM in legs; and $\mathrm{aFM} / \mathrm{BMI}$ ratio.

\subsection{DPN Evaluation by SUDOSCAN}

In order to assess sudomotor function, SUDOSCAN ${ }^{\circledR}$ device (Impeto Medical; Paris, France) was applied for electrochemical skin conductance (ESC) measurements from hands and the feet (both right and left sides). The procedure described in previous studies [33] was followed by trained staff, and results were obtained. The average of the right and left FESC and hand electrochemical skin conductance (HESC) values were used for statistical analysis, while feet asym- 
metry (FASYM, \%) and hands asymmetry (HASYM, \%) values were not included.

\subsection{Statistics}

SPSS 26.0 software (SPSS, Inc., Chicago, IL, USA) was used for all statistical analyses. For normally distributed and skewed quantitative variables, all data were presented as the mean \pm SD or median (inter-quartile range). Shapiro-Wilk test was applied to analyze the normality of the distribution of continuous variables. Dichotomous variables were presented as percentages and were compared using the Chi-square test. Non-parametric variables were compared using the Kruskal-Wallis test, while the One Way ANOVA test was employed to compare normally distributed data between groups. The relationships between ESC in feet and other variables were analyzed using Spearman's correlation analysis. Different linear regression models (Model 1 is unadjusted; Model 2 is adjusted for age and diabetes duration; Model 3 is adjusted for age, diabetes duration, WC, systolic BP, HbA1c, FPG, HOMA-IR; and Model 4 is adjusted for age, diabetes duration, WC, systolic BP, HbAlc, FPG, HOMA-IR, ESR, HDL-C, LDL-C, TC, ALT were used to assess relationships between ESC in feet and various body composition parameters. P-value $<0.05$ was considered statistically significant across all analyses.

\section{Results}

\subsection{Demographic, Lifestyle and Clinical Parameters of the Three Groups}

Among 136 T2DM subjects, 52.2\% were males, 29.4\% were current smokers, $25.7 \%$ were alcohol consumers, median diabetes duration was 12 (6 - 19) years, and mean age was $60.4 \pm 10.1$ years. For biochemical parameters, the average of $\mathrm{HbA1c}$ and TyG indices were $8.77 \% \pm 1.85$ and $4.85 \pm 0.3$, respectively. The median LAP was 45.4 (33.3 - 72.8), and the median VAI was 98.9 (67.3 - 164.4). Hypertension was the most prevalent among present co-morbidities accounting for $69.1 \%$, while diabetic retinopathy was present in $52.9 \%$ of subjects. No significant differences were observed for compared parameters between groups, except for the presence of retinopathy ( $<<0.03)$, BMI ( $<0.012$ ), waist circumference $(\mathrm{p}<0.05)$ and HESC $(\mathrm{p}<0.05)$. The basic characteristics of subjects are illustrated in Table 1.

In terms of BCA parameters, the significant differences were found in $\mathrm{TF}(\mathrm{p}=$ $0.023), \% \mathrm{TF}(\mathrm{p}=0.025), \% \mathrm{AF}(\mathrm{p}=0.048), \mathrm{FM}$ in arms $(\mathrm{p}=0.016), \mathrm{FM}$ in legs ( $\mathrm{p}$ $=0.002), a F M(p=0.002), a F M / B M I$ ratio $(p=0.009)$ between three groups. The BCA parameters of patients are presented in Table 2.

\subsection{Clinical Parameters Associated with FESC (Feet Electrochemical Skin Conductance)}

According to Spearman correlation analysis, FESC was positively correlated with 
Table 1. Baseline parameters of the study population.

\begin{tabular}{|c|c|c|c|c|c|}
\hline Parameters & All $(\mathrm{n}=136)$ & $\begin{array}{c}\text { Group } 1(\mathrm{n}=51) \\
\text { FESC }>70 \mu \mathrm{S}\end{array}$ & $\begin{array}{l}\text { Group } 2(n=46) \\
\text { FESC } 50-70 \mu S\end{array}$ & $\begin{array}{c}\text { Group } 3(n=39) \\
\text { FESC }<50 \mu S\end{array}$ & P-value \\
\hline Age, (years) & $60.4 \pm 10.1$ & $58.4 \pm 11.8$ & $61.4 \pm 7.82$ & $62.1 \pm 9.68$ & 0.168 \\
\hline Male, n \% & 52.2 & 43.1 & 54.3 & 61.5 & 0.209 \\
\hline DM duration, (years) & $12.0(6.0-19.0)$ & $10(2.0-17.0)$ & $12.0(7.0-17.5)$ & $15.0(7.0-20.0)$ & 0.197 \\
\hline Smoker, n \% & 29.4 & 25.0 & 24.1 & 48.1 & 0.065 \\
\hline Drinker, n \% & 25.7 & 15.7 & 26.1 & 35.7 & 0.050 \\
\hline Hypertension, \% & 69.1 & 64.7 & 73.9 & 69.2 & 0.619 \\
\hline CVD, $\%$ & 35.3 & 31.4 & 32.6 & 43.6 & 0.435 \\
\hline Osteoporosis, \% & 11.1 & 7.8 & 13.3 & 12.8 & 0.640 \\
\hline Dyslipidemia, \% & 66.9 & 66.7 & 67.4 & 66.7 & 0.996 \\
\hline Diabetic Retinopathy, \% & 52.9 & 41.2 & 52.2 & 69.2 & 0.030 \\
\hline OADD, \% & 57.4 & 60.8 & 60.9 & 48.7 & 0.435 \\
\hline OADD + Insulin, $\%$ & 42.6 & 39.2 & 39.1 & 51.3 & 0.435 \\
\hline Antilipidemic, \% & 66.9 & 66.7 & 67.4 & 66.7 & 0.996 \\
\hline Antihypertensive, $\%$ & 69.1 & 64.7 & 73.9 & 69.2 & 0.619 \\
\hline Aspirin, \% & 60.3 & 52.9 & 65.2 & 64.1 & 0.396 \\
\hline Beta-blockers, \% & 25.7 & 25.5 & 28.3 & 23.1 & 0.861 \\
\hline BMI $\left(\mathrm{kg} / \mathrm{m}^{2}\right)$ & $25.3(23.2-27.8)$ & $26.6(24.2-29.6)$ & $24.6(22.7-27.2)$ & $25.1(22.6-27.9)$ & 0.012 \\
\hline $\mathrm{WC}(\mathrm{cm})$ & $95.0 \pm 10.1$ & $98.1 \pm 9.30$ & $92.7 \pm 9.1$ & $93.8 \pm 11.2$ & 0.019 \\
\hline Height $(\mathrm{cm})$ & $166.3 \pm 7.38$ & $165.5 \pm 7.43$ & $166.7 \pm 6.82$ & $166.8 \pm 8.03$ & 0.646 \\
\hline Systolic BP (mm Hg) & $137.7 \pm 18.7$ & $137.6 \pm 19.2$ & $137.8 \pm 20.0$ & $137.6 \pm 16.9$ & 0.998 \\
\hline Diastolic BP (mm Hg) & $79.2 \pm 11.6$ & $79.9 \pm 13.3$ & $78.6 \pm 9.63$ & $79.0 \pm 11.5$ & 0.864 \\
\hline $\operatorname{HESC}(\mu S)$ & $64.0(53.2-75.0)$ & $74.0(63.0-79.0)$ & $63.0(53.7-74.0)$ & $49.0(25.0-64.0)$ & 0.001 \\
\hline TyG & $4.85 \pm 0.3$ & $4.87 \pm 0.29$ & $4.81 \pm 0.32$ & $4.85 \pm 0.26$ & 0.594 \\
\hline LAP & $45.4(33.3-72.8)$ & $54.9(37.7-86.4)$ & $42.8(27.5-72.5)$ & $42.7(25.0-67.9)$ & 0.091 \\
\hline VAI & $98.9(67.3-164.4)$ & $121.4(69.0-170.9)$ & $89.4(66.5-150.9)$ & $97.8(65.1-138.9)$ & 0.251 \\
\hline Chinese VAI & $131.9 \pm 37.9$ & $139.9 \pm 35.3$ & $124.5 \pm 33.1$ & $130.5 \pm 45.0$ & 0.133 \\
\hline Hemoglobin (g/l) & $137.3 \pm 14.6$ & $138.6 \pm 13.4$ & $136.1 \pm 14.5$ & $137.0 \pm 16.3$ & 0.695 \\
\hline Red Blood Cells $\left(10^{12} / \mathrm{L}\right)$ & $4.56(4.26-4.83)$ & $4.65(4.31-4.86)$ & $4.56(4.24-4.85)$ & $4.43(4.22-4.82)$ & 0.233 \\
\hline $\operatorname{ESR}(\mathrm{mm} / \mathrm{h})$ & $18.0(9.0-30.0)$ & $15.0(8.0-27.0)$ & $20.0(9.0-27.2)$ & $19.0(11.0-33.0)$ & 0.314 \\
\hline $\mathrm{FPG}(\mathrm{mmol} / \mathrm{l})$ & $6.89(5.73-8.61)$ & $7.09(5.92-8.62)$ & $6.75(5.28-7.99)$ & $7.10(5.91-9.60)$ & 0.244 \\
\hline HbAlc (\%) & $8.77 \pm 1.85$ & $8.96 \pm 1.90$ & $8.36 \pm 1.96$ & $9.01 \pm 1.60$ & 0.175 \\
\hline HOMA-IR & $3.89(2.37-6.85)$ & $4.09(2.73-7.04)$ & $3.24(1.96-6.76)$ & $3.60(2.21-6.22)$ & 0.498 \\
\hline eGFR $\left(\mathrm{ml} / \mathrm{min} / 1.73 \mathrm{~m}^{2}\right)$ & $97.2(91.8-106.1)$ & $98.5(94.7-109.9)$ & $96.2(90.2-104.2)$ & $97.2(91.1-103.1)$ & 0.193 \\
\hline HDL-C (mmol/l) & $1.07(0.92-1.33)$ & $1.08(0.94-1.32)$ & $1.10(0.94-1.35)$ & $1.03(0.89-1.37)$ & 0.651 \\
\hline LDL-C (mmol/l) & $2.74 \pm 0.87$ & $2.97 \pm 0.90$ & $2.63 \pm 0.86$ & $2.58 \pm 0.81$ & 0.065 \\
\hline Triglycerides (mmol/l) & $1.38(1.06-1.94)$ & $1.44(1.13-2.16)$ & $1.26(1.03-2.13)$ & $1.01(1.45-1.86)$ & 0.754 \\
\hline $\mathrm{TC}(\mathrm{mmol} / \mathrm{l})$ & $4.50 \pm 1.09$ & $4.72 \pm 1.10$ & $4.46 \pm 1.08$ & $4.27 \pm 1.07$ & 0.144 \\
\hline Homocysteine $(\mu \mathrm{mol} / \mathrm{L})$ & $11.5(9.5-14.3)$ & $10.5(8.8-13.5)$ & $12.2(10.1-14.4)$ & $11.6(9.3-15.3)$ & 0.051 \\
\hline
\end{tabular}




\section{Continued}

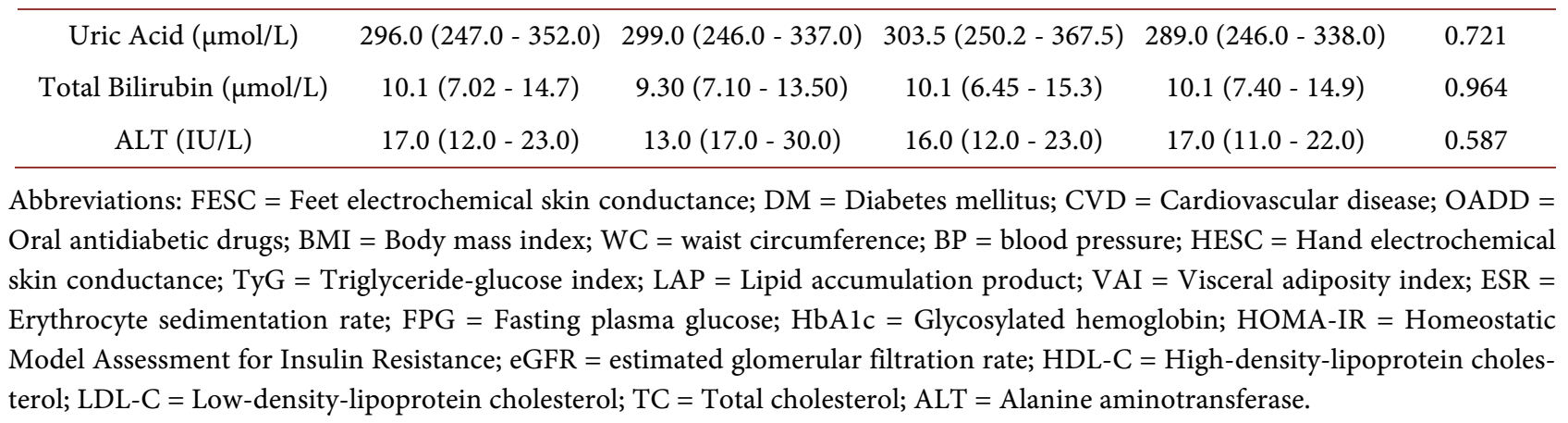

Table 2. Body composition analysis (BCA) parameters of participants.

\begin{tabular}{cccccc}
\hline BCA Parameters & All $(\mathrm{n}=136)$ & $\begin{array}{c}\text { Group } 1(\mathrm{n}=51) \\
\text { FESC }>70 \mu \mathrm{S}\end{array}$ & $\begin{array}{c}\text { Group } 2(\mathrm{n}=46) \\
\text { FESC } 50-70 \mu \mathrm{S}\end{array}$ & $\begin{array}{c}\text { Group } 3(\mathrm{n}=39) \\
\text { FESC }<50 \mu S\end{array}$ \\
\hline TF $(\mathrm{kg})$ & $22.4(18.4-26.4)$ & $23.0(20.3-30.4)$ & $21.3(18.3-24.4)$ & $22.9(16.5-25.7)$ & 0.023 \\
\% TF & $32.3 \pm 6.32$ & $34.2 \pm 6.79$ & $31.2 \pm 5.83$ & $31.1 \pm 5.77$ & 0.025 \\
\%AF & $36.3 \pm 6.66$ & $38.1 \pm 6.62$ & $35.4 \pm 6.62$ & $35.1 \pm 6.40$ & 0.048 \\
TLM (kg) & $46.2(40.6-53.0)$ & $47.7(40.2-52.9)$ & $44.4(40.5-54.7)$ & $47.2(41.2-52.5)$ & 0.958 \\
LM in arms $(\mathrm{kg})$ & $4.72(3.94-5.68)$ & $4.72(3.92-5.74)$ & $4.76(3.95-5.70)$ & $4.67(3.83-5.35)$ & 0.872 \\
LM in legs $(\mathrm{kg})$ & $13.5(11.7-16.1)$ & $13.8(11.9-16.1)$ & $13.3(11.9-16.4)$ & $13.5(11.4-15.6)$ & 0.797 \\
FM in arms $(\mathrm{kg})$ & $2.80(2.27-3.39)$ & $2.97(2.47-4.01)$ & $2.74(2.09-3.25)$ & $2.51(1.97-3.27)$ & 0.016 \\
FM in legs $(\mathrm{kg})$ & $5.65(4.28-7.20)$ & $6.40(4.79-8.10)$ & $5.48(4.19-6.43)$ & $4.94(3.99-6.48)$ & 0.002 \\
aFM $(\mathrm{kg})$ & $8.5(6.55-10.3)$ & $9.08(7.73-12.1)$ & $8.22(6.33-9.65)$ & $7.46(5.92-9.51)$ & 0.002 \\
aLM $(\mathrm{kg})$ & $18.1(15.9-21.8)$ & $18.3(16.0-21.9)$ & $17.8(16.0-22.6)$ & $18.4(15.3-20.9)$ & 0.820 \\
aLM/BMI ratio & $0.71(0.62-0.85)$ & $0.66(0.60-0.81)$ & $0.76(0.67-0.86)$ & $0.72(0.62-0.86)$ & 0.075 \\
aFM/BMI ratio & $0.33(0.27-0.39)$ & $0.37(0.29-0.43)$ & $0.33(0.26-0.38)$ & $0.30(0.26-0.36)$ & 0.009 \\
RSMI $\left(\mathrm{kg} / \mathrm{m}^{2}\right)$ & $6.74(6.03-7.54)$ & $6.93(6.16-7.64)$ & $6.58(6.05-7.58)$ & $6.58(5.96-7.23)$ & 0.374 \\
\hline
\end{tabular}

Abbreviations: FESC $=$ Feet electrochemical skin conductance; $\mathrm{TF}=$ Total fat AF = Android fat; $\mathrm{TLM}=\mathrm{Total}$ lean mass; $\mathrm{LM}=$ lean mass; FM = fat mass; aFM = appendicular FM; aLM = appendicular lean mass; $\mathrm{BMI}=$ body mass index; RSMI = relative skeletal muscle index.

RBC $(\mathrm{r}=0.171, \mathrm{p}=0.047)$ and HESC $(\mathrm{r}=0.569, \mathrm{p}=0.0001)$, although negative correlations were also observed for ESR and homocysteine $(r=-0.190, r=$ $-0.192, \mathrm{p}<0.05)$, respectively. No significant correlations between FESC and other variables were observed (Table 3 ).

\subsection{Associations of BCA Parameters with FESC}

FESC was positively correlated with both FM in arms $(r=0.205, \mathrm{p}<0.017)$, and $\mathrm{FM}$ in legs $(\mathrm{r}=0.225, \mathrm{p}<0.008)$. Moreover, significant positive correlations were observed between FESC and newly proposed BCA indices, such as aFM and aFM/BMI ratio $(\mathrm{r}=0.006, \mathrm{r}=0.012, \mathrm{p}<0.05)$, respectively. However, there were no significant correlations between FESC and muscle mass-related BCA parameters (TLM, aLM, aLM/BMI ratio, etc.) (Table 3). 
Table 3. The results of Spearman correlation analysis between various parameters and FESC.

\begin{tabular}{|c|c|c|}
\hline \multirow{2}{*}{ Variables } & \multicolumn{2}{|c|}{ FESC } \\
\hline & Correlation coefficient & $P$ value \\
\hline $\mathrm{TF}(\mathrm{kg})$ & 0.144 & 0.094 \\
\hline$\% \mathrm{TF}$ & 0.167 & 0.051 \\
\hline$\% \mathrm{AF}$ & 0.123 & 0.155 \\
\hline TLM (kg) & 0.004 & 0.968 \\
\hline $\mathrm{LM}$ in arms $(\mathrm{kg})$ & 0.031 & 0.724 \\
\hline LM in legs $(\mathrm{kg})$ & 0.037 & 0.668 \\
\hline FM in arms $(\mathrm{kg})$ & 0.205 & 0.017 \\
\hline FM in legs $(\mathrm{kg})$ & 0.225 & 0.008 \\
\hline $\mathrm{aFM}(\mathrm{kg})$ & 0.234 & 0.006 \\
\hline aLM (kg) & 0.036 & 0.675 \\
\hline aLM/BMI ratio & -0.101 & 0.244 \\
\hline $\mathrm{aFM} / \mathrm{BMI}$ ratio & 0.216 & 0.012 \\
\hline $\operatorname{RSMI}\left(\mathrm{kg} / \mathrm{m}^{2}\right)$ & 0.098 & 0.258 \\
\hline BMI $\left(\mathrm{kg} / \mathrm{m}^{2}\right)$ & 0.167 & 0.053 \\
\hline $\mathrm{WC}(\mathrm{cm})$ & 0.110 & 0.202 \\
\hline TyG & 0.014 & 0.873 \\
\hline LAP & 0.134 & 0.121 \\
\hline VAI & 0.096 & 0.268 \\
\hline Chinese VAI & 0.048 & 0.578 \\
\hline $\operatorname{HESC}(\mu S)$ & 0.569 & 0.0001 \\
\hline Hemoglobin (g/l) & 0.114 & 0.185 \\
\hline Red Blood Cells $\left(10^{12} / \mathrm{L}\right)$ & 0.171 & 0.047 \\
\hline $\operatorname{ESR}(\mathrm{mm} / \mathrm{h})$ & -0.190 & 0.027 \\
\hline FPG (mmol/l) & -0.030 & 0.729 \\
\hline HbAlc (\%) & 0.028 & 0.743 \\
\hline eGFR (ml/min) & 0.141 & 0.102 \\
\hline HDL-C (mmol/l) & 0.060 & 0.487 \\
\hline $\mathrm{LDL}-\mathrm{C}(\mathrm{mmol} / \mathrm{l})$ & 0.128 & 0.138 \\
\hline Triglyceride (mmol/l) & 0.030 & 0.728 \\
\hline $\mathrm{TC}(\mathrm{mmol} / \mathrm{l})$ & 0.148 & 0.085 \\
\hline Homocysteine $(\mu \mathrm{mol} / \mathrm{L})$ & -0.192 & 0.025 \\
\hline Total Bilirubin $(\mu \mathrm{mol} / \mathrm{L})$ & 0.029 & 0.734 \\
\hline $\operatorname{ALT}(\mathrm{IU} / \mathrm{L})$ & 0.047 & 0.586 \\
\hline
\end{tabular}

Abbreviations: FESC = Feet electrochemical skin conductance; $\mathrm{TF}=$ Total fat; $\mathrm{AF}=\mathrm{An}$ droid fat; TLM = Total lean mass; $L M=$ lean mass; $F M=$ fat mass; $\mathrm{aFM}=$ appendicular FM; aLM = appendicular lean mass; $\mathrm{BMI}$ = body mass index; RSMI = relative skeletal muscle index; $\mathrm{BMI}=$ Body mass index; $\mathrm{WC}=$ waist circumference; $\mathrm{TyG}=$ Triglyceride-glucose index; LAP = Lipid accumulation product; VAI = Visceral adiposity index; $\mathrm{DM}=$ Diabetes mellitus; HESC $=$ Hand electrochemical skin conductance; ESR = Erythrocyte sedimentation rate; FPG = Fasting plasma glucose;HbA1c = Glycosylated hemoglobin; eGFR = estimated glomerular filtration rate; HDL-C = High-density-lipoprotein cholesterol; LDL-C = Low-density-lipoprotein cholesterol; $\mathrm{TC}=$ Total cholesterol; ALT = Alanine aminotransferase. 
To further assess their independent associations with FESC, those BCA parameters significantly correlated with FESC in Spearman correlation were analyzed in multivariate linear regression. In Model 2 (adjusted for age and diabetes duration) and Model 3 (further adjusted for WC, systolic BP, HbAlc, FPG, HOMA-IR), FM in arms, FM in legs, aFM and aFM/BMI ratio were independently associated with FESC $(\beta=0.201,0.190,0.204,0.205, \mathrm{p}<0.05$, respectively). Moreover, even after further adjustments for ESR, HDL-C, LDL-C, Total Cholesterol, ALT, the same BCA parameters remained independently associated with FESC (Table 4). None of the other well-known indices (TyG, LAP, VAI and Chinese VAI) was significantly associated with FESC (data are not illustrated).

\section{Discussion}

The fat distribution has a significant influence on DPN development and progression. As the primary source of anti-inflammatory adipokines such as leptin and adiponectin, subcutaneous fat deposition is accompanied by a better lipid profile and improved glucose control [34]. Especially gluteofemoral subcutaneous

Table 4. Association of FESC with BCA parameters by univariate and multivariate linear regression analysis.

\begin{tabular}{|c|c|c|c|c|c|c|c|c|c|c|c|c|}
\hline \multirow{2}{*}{ Variables } & \multicolumn{3}{|c|}{ MODEL 1} & \multicolumn{3}{|c|}{ MODEL 2} & \multicolumn{3}{|c|}{ MODEL 3} & \multicolumn{3}{|c|}{ MODEL 4} \\
\hline & $\beta$ & $\mathrm{t}$ & $P$ value & $\beta$ & $\mathrm{t}$ & $P$ value & $\beta$ & $\mathrm{t}$ & $\mathrm{P}$ value & $\beta$ & $\mathrm{t}$ & $P$ value \\
\hline $\mathrm{TF}(\mathrm{kg})$ & 0.225 & 2.667 & 0.009 & 0.13 & 1.499 & 0.136 & 0.081 & 1.219 & 0.225 & 0.099 & 1.446 & 0.151 \\
\hline$\% \mathrm{TF}$ & 0.132 & 1.536 & 0.127 & 0.172 & 2.05 & 0.042 & 0.153 & 1.894 & 0.061 & 0.141 & 1.782 & 0.077 \\
\hline$\% \mathrm{AF}$ & 0.124 & 1.445 & 0.151 & 0.142 & 1.66 & 0.099 & 0.112 & 1.430 & 0.155 & 0.091 & 1.102 & 0.273 \\
\hline TLM (kg) & 0.162 & 1.897 & 0.06 & -0.05 & -0.604 & 0.547 & -0.093 & -1.29 & 0.196 & -0.071 & -1.034 & 0.303 \\
\hline LM in arms $(\mathrm{kg})$ & 0.178 & 2.091 & 0.038 & 0.006 & 0.069 & 0.945 & -0.031 & -0.403 & 0.688 & -0.002 & -0.031 & 0.975 \\
\hline LM in legs $(\mathrm{kg})$ & 0.016 & 0.191 & 0.849 & -0.035 & -0.418 & 0.676 & -0.074 & -0.960 & 0.339 & -0.044 & -0.572 & 0.569 \\
\hline FMin arms $(\mathrm{kg})$ & 0.204 & 2.412 & 0.017 & 0.201 & 2.346 & 0.020 & 0.161 & 2.162 & 0.032 & 0.167 & 2.287 & 0.024 \\
\hline FM in legs $(\mathrm{kg})$ & 0.198 & 2.337 & 0.021 & 0.19 & 2.227 & 0.028 & 0.155 & 1.990 & 0.049 & 0.182 & 2.346 & 0.021 \\
\hline aFM (kg) & 0.210 & 2.489 & 0.014 & 0.204 & 2.392 & 0.018 & 0.165 & 2.188 & 0.030 & 0.240 & 2.979 & 0.013 \\
\hline aLM (kg) & 0.03 & 0.343 & 0.732 & -0.024 & -0.296 & 0.767 & -0.064 & -0.839 & 0.403 & -0.033 & -0.451 & 0.653 \\
\hline aLM/BMI ratio & -0.082 & -0.956 & 0.341 & -0.116 & -1.36 & 0.176 & -0.121 & -1.42 & 0.158 & -0.103 & -1.253 & 0.212 \\
\hline aFM/BMI ratio & 0.189 & 2.223 & 0.028 & 0.205 & 2.430 & 0.016 & 0.185 & 2.214 & 0.029 & 0.214 & 2.642 & 0.022 \\
\hline $\operatorname{RSMI}\left(\mathrm{kg} / \mathrm{m}^{2}\right)$ & 0.088 & 1.028 & 0.306 & 0.032 & 0.386 & 0.702 & -0009 & -0116 & 0.908 & 0.027 & 0.361 & 0.719 \\
\hline
\end{tabular}

Model 1, Unadjusted; Model 2, adjusted for Age and DM duration; Model 3, adjusted for Age, DM duration, WC, systolic BP, HbA1c, FPG, HOMA-IR; Model 4, adjusted for Age, DM duration, WC, systolic BP, HbA1c, FPG, HOMA-IR, ESR, HDL-C, LDL-C, TC, ALT; Abbreviations: TF = Total fat; AF = Android fat; TLM = Total lean mass; LM = lean mass; FM = fat mass; aFM = appendicular FM; aLM = appendicular lean mass; BMI = body mass index; RSMI = relative skeletal muscle index; DM = diabetes mellitus; $\mathrm{WC}=$ waist circumference; $\mathrm{BP}=$ blood pressure; HbA1c = Glycosylated hemoglobin; FPG = Fasting plasma glucose; HOMA-IR = Homeostatic Model Assessment for Insulin Resistance; ESR = Erythrocyte sedimentation rate; HDL-C = High-density-lipoprotein cholesterol; LDL-C = Low-density-lipoprotein cholesterol; TC = Total cholesterol; ALT = Alanine aminotransferase. 
fat indirectly decreases lipotoxicity and prevents excess ectopic fat accumulation, particularly in intermuscular compartments [35] [36]. Such protective properties of subcutaneous fat can be explained by its preferential intake of excess free fatty acid (FFA) levels that prevent muscle tissue from ectopic fat accumulation [35] [37].

To our knowledge, it is a pioneering study conducted to evaluate the association between sudomotor dysfunction and various anthropometric measurements, especially newly proposed parameters including aFM, aFM/BMI ratio. The current study revealed an association between DPN, assessed by sudomotor function, and leg subcutaneous fat. Similarly, such a relationship was highlighted previously [38] [39]. Zhen et al. (2018) suggested that the rise of leg subcutaneous fat for $1 \mathrm{~kg}$ results in a 30\% reduction of DPN likelihood. In addition to this, Bittel et al. (2015) has found that diabetic patients without DPN have more subcutaneous and less intermuscular deposition of fat than counterparts with DPN. He also proposed that the transition from T2DM to T2DM with DPN may be accompanied by a progressive shift of subcutaneous fat deposition towards intermuscular compartments. Such a gradual rise in fat deposition in the intermuscular region may lead to excess cytokines secretion [40], resulting in further degenerative changes of nerve fibers [41]. It is well studied that $\beta$-oxidation of excessive FFA by the nerve structures during hyperlipidemic state leads to Schwann cells damage via increased reactive oxygen substrates (ROS) generation [42]. Additionally, local rise of cholesterol concentration occurring in ectopic fat accumulation state results in increased oxysterols levels that contributes to neuronal damage [43]. Moreover, intermuscular fat volume was negatively correlated with both muscle strength and performance [39]. However, in the studies mentioned above [38] [39], DPN diagnosis was confirmed through nerve conduction studies or MNSI questionnaires. Our results suggest that such fat redistribution may be present even in the early stages of DPN characterized by sudomotor dysfunction. According to some authors [44], the shift in fat deposition from the subcutaneous region towards intermuscular is age-related. Nonetheless, even after adjustment for age, a decline in FESC was still correlated with subcutaneous fat loss in our research. The present study's findings may further confirm the protective role of subcutaneous fat in the progression of DPN. In addition to this, we also found similar associations between FESC and fat in arms, aFM, aFM/BMI ratio. However, ongoing studies are required to further investigate the predictive value of these novel indices in both healthy and diabetic individuals.

Our study also revealed that the progression of DPN has been associated with a decrease in neither aLM nor aLM/BMI ratio. Interestingly, the mean aLM results of patients with severely reduced FESC were higher than those of those with mildly reduced FESC. Possibly, aLM increased due to excess fat accumulation, not because of muscle hypertrophy in the current study. It was also confirmed in The Health ABC study that appendicular muscle mass does not accurately reflect the muscle strength in elderly diabetic patients [8]. Moreover, di- 
abetic patients had more appendicular muscle mass than non-diabetic participants in the same study.

We found that sudomotor dysfunction was significantly associated with the presence of retinopathy. Similarly, previous studies have explored this relationship, where sudomotor dysfunction was assessed using different techniques [45] [46]. It can be explained by the fact that sudomotor dysfunction is associated with microvascular complications [47]. The inverse association between sudomotor dysfunction and total bilirubin levels in type 2 diabetic patients has been found previously [48], although no relationship has been revealed in the current study.

We finally investigated the relationship between sudomotor dysfunction and various anthropo-metabolic indices. Noticeably, no associations were identified between sudomotor dysfunction and commonly used anthropo-metabolic indices, such as TyG index, VAI, LAP and Chinese VAI. Although these indices have already been considered useful predictors of different metabolic alterations [47] [48], their importance in early DPN evaluation seems limited. Since several studies have revealed conflicting results regarding the relationship between lipid profile parameters and DPN [49] [50], our findings supporting no association between those factors should be carefully interpreted.

The current study has some limitations. Firstly, this investigation was monocentric. We also consider the relatively small number of participants as another limitation, although each investigated group had a similar number of subjects. Besides this, we proposed that excess intermuscular fat deposition increased appendicular muscle mass, although we did not clarify it by using MRI because of its high cost. Since we aimed to investigate the early stage of DPN and, even more, nerve conduction studies were not available for a significant number of patients, we used only SUDOSCAN results for DPN assessment. Nonetheless, SUDOSCAN has already been considered an effective DPN detection tool with high sensitivity and specificity [18].

\section{Conclusion}

In conclusion, the present pioneering study revealed that progressive decline of sudomotor function is positively associated with loss of subcutaneous fat in the extremities. Therefore, it is reasonable to suggest that a decrease of subcutaneous fat in extremities may even be present in the early stages of DPN in type 2 diabetic patients. Since aFM and aFM/BMI ratios were used for the first time in the current study, future studies should investigate their importance. Further studies are needed for a comprehensive understanding of this relationship and exploration of the importance of subcutaneous fat as a protective factor against DPN progression.

\section{Funding Statement}

This research did not receive any specific grant from funding agencies in the 
public, commercial, or not-for-profit sectors.

\section{CRediT Authorship Contribution Statement}

Bekzod Odilov: Conceptualization, Methodology, Writing-original draft, Investigation, Validation. Danfeng Yu: Resources, Data Curation. Amir Musa Mohamud: Software, Formal analysis, Validation. Ruxing Zhao: Resources. Ying Zou: Writing-Review \& editing, Visualization. Xinguo Hou: Conceptualization, Methodology, Validation, Resources, Writing-Review \& editing, Supervision, Project administration.

\section{Acknowledgements}

The authors are thankful to Ahmed Badughaish for his proofreading assistance.

\section{Conflicts of Interest}

The authors reported no conflict of interest.

\section{References}

[1] Saeedi, P., Petersohn, I., Salpea, P., et al. (2019) Global and Regional Diabetes Prevalence Estimates for 2019 and Projections for 2030 and 2045: Results from the International Diabetes Federation Diabetes Atlas, 9th Edition. Diabetes Research and Clinical Practice, 157, Article ID: 107843. https://doi.org/10.1016/j.diabres.2019.107843

[2] Dyck, P.J., Kratz, K.M., Karnes, J.L., et al. (1993) The Prevalence by Staged Severity of Various Types of Diabetic Neuropathy, Retinopathy, and Nephropathy in a Population-Based Cohort: The Rochester Diabetic Neuropathy Study. Neurology, 43, 817-824. https://doi.org/10.1212/WNL.43.4.817

[3] Gordois, A., Scuffham, P., Shearer, A., et al. (2003) The Health Care Costs of Diabetic Peripheral Neuropathy in the US. Diabetes Care, 26, 1790-1795. https://doi.org/10.2337/diacare.26.6.1790

[4] Argoff, C.E., Cole, B.E., Fishbain, D.A., et al. (2006) Diabetic Peripheral Neuropathic Pain: Clinical and Quality-of-Life Issues. Mayo Clinic Proceedings, 81, S3-S11. https://doi.org/10.1016/S0025-6196(11)61474-2

[5] Boyle, J.P., Thompson, T.J., Gregg, E.W., et al. (2010) Projection of the Year 2050 Burden of Diabetes in the US Adult Population: Dynamic Modeling of Incidence, Mortality, and Prediabetes Prevalence. Population Health Metrics, 8, 29. https://doi.org/10.1186/1478-7954-8-29

[6] Larsson, L., Degens, H., Li, M., et al. (2019) Sarcopenia: Aging-Related Loss of Muscle Mass and Function. Physiological Reviews, 99, 427-511. https://doi.org/10.1152/physrev.00061.2017

[7] Delmonico, M.J., Harris, T.B., Lee, J.-S., et al. (2007) Alternative Definitions of Sarcopenia, Lower Extremity Performance, and Functional Impairment with Aging in Older men and Women. Journal of the American Geriatrics Society, 55, 769-774. https://doi.org/10.1111/j.1532-5415.2007.01140.x

[8] Park, S.W., Goodpaster, B.H., Strotmeyer, E.S., et al. (2006) Decreased Muscle Strength and Quality in Older Adults with Type 2 Diabetes: The Health, Aging, and Body Composition Study. Diabetes, 55, 1813-1818. 
https://doi.org/10.2337/db05-1183

[9] Cruz-Jentoft, A.J., Baeyens, J.P., Bauer, J.M., et al. (2010) Sarcopenia: European Consensus on Definition and Diagnosis. Age Ageing, 39, 412-423.

https://doi.org/10.1093/ageing/afq034

[10] Wang, M., Tan, Y., Shi, Y., et al. (2020) Diabetes and Sarcopenic Obesity: Pathogenesis, Diagnosis, and Treatments. Frontiers in Endocrinology (Lausanne), 11, 568. https://doi.org/10.3389/fendo.2020.00568

[11] Kim, K.-S., Park, K.-S., Kim, M.-J., et al. (2014) Type 2 Diabetes Is Associated with Low Muscle Mass in Older Adults. Geriatrics \& Gerontology International, 14, 115-121. https://doi.org/10.1111/ggi.12189

[12] Ferrari, S.L., Abrahamsen, B., Napoli, N., et al. (2018) Diagnosis and Management of Bone Fragility in Diabetes: An Emerging Challenge. Osteoporosis International, 29, 2585-2596. https://doi.org/10.1007/s00198-018-4650-2

[13] Jang, H.C. (2016) Sarcopenia, Frailty, and Diabetes in Older Adults. Diabetes \& Metabolism Journal, 40, 182-189. https://doi.org/10.4093/dmj.2016.40.3.182

[14] Andersen, H., Gjerstad, M.D. and Jakobsen, J. (2004) Atrophy of Foot Muscles: A Measure of Diabetic Neuropathy. Diabetes Care, 27, 2382-2385. https://doi.org/10.2337/diacare.27.10.2382

[15] Nomura, T., Kawae, T., Kataoka, H., et al. (2018) Assessment of Lower Extremity Muscle Mass, Muscle Strength, and Exercise Therapy in Elderly Patients with Diabetes Mellitus. Environmental Health and Preventive Medicine, 23, 1-7. https://doi.org/10.1186/s12199-018-0710-7

[16] Green, A.Q., Krishnan, S., Finucane, F.M., et al. (2010) Altered C-Fiber Function as an Indicator of Early Peripheral Neuropathy in Individuals with Impaired Glucose Tolerance. Diabetes Care, 33, 174-176. https://doi.org/10.2337/dc09-0101

[17] Kazamel, M., Stino, A.M. and Smith, A.G. (2021) Metabolic Syndrome and Peripheral Neuropathy. Muscle and Nerve, 63, 285-293.

https://doi.org/10.1002/mus.27086

[18] Selvarajah, D., Cash, T., Davies, J., et al. (2015) SUDOSCAN: A Simple, Rapid, and Objective Method with Potential for Screening for Diabetic Peripheral Neuropathy. PLoS ONE, 10, e0138224. https://doi.org/10.1371/journal.pone.0138224

[19] Krieger, S.-M., Reimann, M., Haase, R., et al. (2018) Sudomotor Testing of Diabetes Polyneuropathy. Frontiers in Neurology, 9, Article No. 803.

https://doi.org/10.3389/fneur.2018.00803

[20] Fung, F.Y., Koh, Y.L.E., Malhotra, R., et al. (2019) Prevalence of and Factors Associated with Sarcopenia among Multi-Ethnic Ambulatory Older Asians with Type 2 Diabetes Mellitus in a Primary Care Setting. BMC Geriatrics, 19, Article No. 122. https://doi.org/10.1186/s12877-019-1137-8

[21] Yang, R., Zhang, Y., Shen, X., et al. (2016) Sarcopenia Associated with Renal Function in the Patients with Type 2 Diabetes. Diabetes Research and Clinical Practice, 118, 121-129. https://doi.org/10.1016/j.diabres.2016.06.023

[22] Chung, H.S., Hwang, S.Y., Choi, J.H., et al. (2018) Effects of Low Muscle Mass on Albuminuria and Chronic Kidney Disease in Patients with Type 2 Diabetes: The Korean Sarcopenic Obesity Study (KSOS). The Journals of Gerontology. Series A, Biological Sciences and Medical Sciences, 73, 386-392.

https://doi.org/10.1093/gerona/glx055

[23] (1999) Glucose Tolerance and Mortality: Comparison of WHO and American Diabetes Association Diagnostic Criteria. The DECODE Study Group. European Di- 
abetes Epidemiology Group. Diabetes Epidemiology: Collaborative Analysis of Diagnostic Criteria in Europe. Lancet (London, England), 354, 617-621. https://doi.org/10.1016/S0140-6736(98)12131-1

[24] Hussein, I.I., Alshammary, S.H.A. and Al-Nimer, M.S.M. (2021) Assessment of Sudomotor Function in Hypertensive with/without Type-2 Diabetes Patients Using SUDOSCAN: An Electrophysiological Study. Clinical Neurophysiology Practice, 6, 22-28. https://doi.org/10.1016/j.cnp.2020.12.001

[25] Luo, Z.X., Zeng, Q., Luo, R., et al. (2015) Relative Contributions of Ectopic Liver and Abdominal Fat Accumulation to Arterial Stiffness. Endocrine Practice, 21, 574-580. https://doi.org/10.4158/EP14526.OR

[26] Simental-Mendía, L.E., Rodríguez-Morán, M. and Guerrero-Romero, F. (2008) The Product of Fasting Glucose and Triglycerides as Surrogate for Identifying Insulin Resistance in Apparently Healthy Subjects. Metabolic Syndrome and Related Disorders, 6, 299-304. https://doi.org/10.1089/met.2008.0034

[27] Amato, M.C., Giordano, C., Galia, M., et al. (2010) Visceral Adiposity Index: A Reliable Indicator of Visceral Fat Function Associated with Cardiometabolic Risk. Diabetes Care, 33, 920-922. https://doi.org/10.2337/dc09-1825

[28] Kahn, H.S. (2005) The "Lipid Accumulation Product" Performs Better than the Body Mass Index for Recognizing Cardiovascular Risk: A Population-Based Comparison. BMC Cardiovascular Disorders, 5, Article No. 26. https://doi.org/10.1186/1471-2261-5-26

[29] Wu, J., Gong, L., Li, Q., et al. (2017) A Novel Visceral Adiposity Index for Prediction of Type 2 Diabetes and Pre-Diabetes in Chinese Adults: A 5-Year Prospective Study. Scientific Reports, 7, Article No. 13784. https://doi.org/10.1038/s41598-017-14251-w

[30] Matthews, D.R., Hosker, J.P., Rudenski, A.S., et al. (1985) Homeostasis Model Assessment: Insulin Resistance and Beta-Cell Function from Fasting Plasma Glucose and Insulin Concentrations in Man. Diabetologia, 28, 412-419. https://doi.org/10.1007/BF00280883

[31] Studenski, S.A., Peters, K.W., Alley, D.E., et al. (2014) The FNIH Sarcopenia Project: Rationale, Study Description, Conference Recommendations, and Final Estimates. The Journals of Gerontology. Series A, Biological Sciences and Medical Sciences, 69, 547-558. https://doi.org/10.1093/gerona/glu010

[32] Baumgartner, R.N., Koehler, K.M., Gallagher, D., et al. (1998) Epidemiology of Sarcopenia among the Elderly in New Mexico. The American Journal of Epidemiology, 147, 755-763. https://doi.org/10.1093/oxfordjournals.aje.a009520

[33] Carbajal-Ramírez, A., Hernández-Domínguez, J.A., Molina-Ayala, M.A., et al. (2019) Early Identification of Peripheral Neuropathy Based on Sudomotor Dysfunction in Mexican Patients with Type 2 Diabetes. BMC Neurology, 19, Article No. 109. https://doi.org/10.1186/s12883-019-1332-4

[34] Snijder, M.B., Visser, M., Dekker, J.M., et al. (2005) Low Subcutaneous Thigh Fat Is a Risk Factor for Unfavourable Glucose and Lipid Levels, Independently of High Abdominal Fat. The Health ABC Study. Diabetologia, 48, 301-308. https://doi.org/10.1007/s00125-004-1637-7

[35] Wronska, A. and Kmiec, Z. (2012) Structural and Biochemical Characteristics of Various White Adipose Tissue Depots. Acta Physiologica, 205, 194-208. https://doi.org/10.1111/j.1748-1716.2012.02409.x

[36] Frayn, K.N. (2002) Adipose Tissue as a Buffer for Daily Lipid Flux. Diabetologia, 45, 1201-1210. https://doi.org/10.1007/s00125-002-0873-y 
[37] Porter, S.A., Massaro, J.M., Hoffmann, U., et al. (2009) Abdominal Subcutaneous Adipose Tissue: A Protective Fat Depot? Diabetes Care, 32, 1068-1075. https://doi.org/10.2337/dc08-2280

[38] Zhen, Q., Yao, N., Chen, X., et al. (2019) Total Body Adiposity, Triglycerides, and Leg Fat Are Independent Risk Factors for Diabetic Peripheral Neuropathy in Chinese Patients with Type 2 Diabetes Mellitus. Endocrine Practice, 25, 270-278. https://doi.org/10.4158/EP-2018-0459

[39] Bittel, D.C., Bittel, A.J., Tuttle, L.J., et al. (2015) Adipose Tissue Content, Muscle Performance and Physical Function in Obese Adults with Type 2 Diabetes Mellitus and Peripheral Neuropathy. Journal of Diabetic Complications, 29, 250-257. https://doi.org/10.1016/j.jdiacomp.2014.11.003

[40] Skundric, D.S. and Lisak, R.P. (2003) Role of Neuropoietic Cytokines in Development and Progression of Diabetic Polyneuropathy: From Glucose Metabolism to Neurodegeneration. Experimental Diabetes Research, 4, 303-312. https://doi.org/10.1155/EDR.2003.303

[41] Stehno-Bittel, L. (2008) Intricacies of Fat. Physical Therapy, 88, 1265-1278. https://doi.org/10.2522/ptj.20080034

[42] Padilla, A., Descorbeth, M., Almeyda, A.L., et al. (2011) Hyperglycemia Magnifies Schwann Cell Dysfunction and Cell Death Triggered by PA-Induced Lipotoxicity. Brain Research, 1370, 64-79. https://doi.org/10.1016/j.brainres.2010.11.013

[43] Jang, E.-R. and Lee, C.S. (2011) 7-Ketocholesterol Induces Apoptosis in Differentiated PC12 Cells via Reactive Oxygen Species-Dependent Activation of NF- $\kappa$ B and Akt Pathways. Neurochemistry International, 58, 52-59. https://doi.org/10.1016/j.neuint.2010.10.012

[44] Garg, A. and Agarwal, A.K. (2009) Lipodystrophies: Disorders of Adipose Tissue Biology. Biochimica et Biophysica Acta, 1791, 507-513. https://doi.org/10.1016/j.bbalip.2008.12.014

[45] Veloso, D.L.C., Nascimento, R.C.G., Leite, E.B., et al. (2020) Predictors of Sudomotor Dysfunction in Patients with Type 1 Diabetes without Clinical Evidence of Peripheral Neuropathy. Diabetes Research and Clinical Practice, 170, Article ID: 108500. https://doi.org/10.1016/j.diabres.2020.108500

[46] Lewis, J.E., Atlas, S.E., Rasul, A., et al. (2017) New Method of Sudomotor Function Measurement to Detect Microvascular Disease and Sweat Gland Nerve or Unmyelinated C Fiber Dysfunction in Adults with Retinopathy. Journal of Diabetes \& Metabolic Disorders, 16, 26. https://doi.org/10.1186/s40200-017-0307-5

[47] Gandecka, A., Araszkiewicz, A., Piłaciński, S., et al. (2017) Evaluation of Sudomotor Function in Adult Patients with Long-Lasting Type 1 Diabetes. Polish Archives of Internal Medicine, 127, 16-24. https://doi.org/10.20452/pamw.3884

[48] Mao, F., Zhu, X., Lu, B., et al. (2018) The Association between Serum Bilirubin Level and Electrochemical Skin Conductance in Chinese Patients with Type 2 Diabetes. International Journal of Endocrinology, 2018, Article ID: 6253170. https://doi.org/10.1155/2018/6253170

[49] Ziegler, D., Rathmann, W., Dickhaus, T., et al. (2008) Prevalence of Polyneuropathy in Pre-Diabetes and Diabetes Is Associated with Abdominal Obesity and Macroangiopathy: The MONICA/KORA Augsburg Surveys S2 and S3. Diabetes Care, 31, 464-469. https://doi.org/10.2337/dc07-1796

[50] Callaghan, B.C., Xia, R., Reynolds, E., et al. (2016) Association between Metabolic Syndrome Components and Polyneuropathy in an Obese Population. JAMA Neurology, 73, 1468-1476. https://doi.org/10.1001/jamaneurol.2016.3745 